\title{
A renal vascular compartment segmentation method based on dynamic contrast-enhanced images
}

\author{
Hong $\mathrm{Li}^{\mathrm{a}}$, Nan $\mathrm{Bao}^{\mathrm{a}}$, Xieping $\mathrm{Xu}^{\mathrm{a}}$, Yaonan Zhang ${ }^{\mathrm{a}}$, Shikai Jin ${ }^{\mathrm{a}}$, Yueming $\mathrm{Jin}^{\mathrm{a}}$ and \\ Haoran Sun ${ }^{\mathrm{b}, *}$ \\ a Sino-Dutch Biomedical and Information Engineering School, Northeastern University, Shenyang, \\ Liaoning, China \\ ${ }^{\mathrm{b}}$ Department of Radiology, Tianjin Medical University General Hospital, Tianjin, China
}

\begin{abstract}
.
BACKGROUND: Kidney function assessment from renography has great potential for clinical diagnosis. Compartment models are the main analytical models in this field and the vascular compartment is the most important one, whether in the twocompartment model or three-compartment model. Currently, there are some published research studies on renal cortex segmentation. However, there are few publications introducing the methods on how to segment the vascular compartment yet.

OBJECTIVE: The objective of this paper is to segment the vascular compartment automatically.

METHODS: This method was tested on multi-phase scan images. A feature image reconstructed from the original images was used to segment the vascular compartment. It used the features of the time-density curve of each voxel in the contrast-enhanced images to distinguish vascular space from other areas.

RESULTS: The segmentation result was evaluated by the renal glomerular filtration rate (GFR) analysis of a two-compartment model with the Patlak-Rutland technique. The dataset contained 11 kidney subjects whose GFR ranged from $19.8 \mathrm{ml} / \mathrm{min}$ to $74.9 \mathrm{ml} / \mathrm{min}$. The results showed that the correlation between reference GFR and model derived GFR was $0.919(P<0.001)$. CONCLUSION: Compared with segmentation performed on certain phase images, this method can avoid the problem of subjective phase selection. For a given kidney data, the proposed method can always obtain the same segmentation result automatically.
\end{abstract}

Keywords: Renal function, GFR, renography, vascular compartment segmentation, time density curve, contrast-enhanced image

\section{Introduction}

The kidney is a vital organ to filter the metabolic waste products and maintain the balance of body fluid. Renal function assessment plays an important role in the diagnosis of kidney diseases. Since the clearance of serum creatinine measurement is imprecise and cannot evaluate single kidney, the assessment based on renography of medical images became a very hot area. In recent years, many methods and models have been proposed by researchers to measure glomerular filtration rate (GFR) and other important parameters of kidney.

\footnotetext{
${ }^{*}$ Corresponding author: Haoran Sun, Department of Radiology, Tianjin Medical University General Hospital, Tianjin, China. Tel.: +86 1382159 2000; E-mail: sunhaoran2006@ hotmail.com.
} 
In an early study by Baumann et al. and Didier et al. [1,2], they defined initial inflow of contrast agent from the cortex into the medulla, the cortex was identified with the vascular space. A parameter $k_{c l}$ was regarded as an estimate for the clearance rate. Hackstein et al. [3] applied a two-compartment model with Patlak plot technique. In this method, two compartments were modeled as the vascular space and the nephron space separately, and the outflow and dispersion of the bolus were ignored. It assumed that unilateral tracer flowed from vascular compartment into nephron compartment [4-6]. However, in the paper of Buckley et al. [7] and Annet et al. [8], those factors were considered individually. Lee et al. [9] proposed a multi-compartmental model which described three compartments including vascular compartment, proximal tubules and loops of Henle. Based on Lee's model, Zhang et al. [10] assumed that due to a distribution of pathways, the contrast took a minimum transit time to traverse through each compartment before emerging at the outlet. Hence, an impulse retention function (IRF) was applied to this model.

Above all theses models, the vascular compartment is one of the most important compartment in which filtration process takes place. Due to lacking the way of segmenting vascular compartment, the contrast concentration in the vascular compartment was always replaced by that in the aorta when using the two-compartment model. However, this substitution will bring errors and will be a major source of uncertainty in GFR measurement. What's more, in three-compartment model, since the vascular compartment is an unknown parameter, the process of solving equation became much more complicated.

Although there are few vascular compartment segmentation methods published yet, the renal cortex and medulla segmentation is common and the methods can be found in some papers. In order to implement the renal function analysis model, manual segmentation of the ROIs can be found in many papers $[3,7,8,11,12]$. Sometimes, automatic or semi-automatic methods were applied to the models. Boykov et al. [13-15] used a semi-automatic algorithm based on interactive graph cuts. Thresholding method was also used to segment the cortex from the kidney [16-18].

The methods talked above were used to segment the renal cortex and medulla from original images. However, the vascular compartment cannot be distinguished directly from the original images. Parts of the vascular compartment are in the cortex and the other parts of it are in the medulla. In this paper, we proposed a method to segment vascular compartment from a feature image which was reconstructed from the original images. The segmentation result was evaluated by the renal glomerular filtration rate (GFR) analysis of a two-compartment model with the Patlak-Rutland technique. Linear regression and correlation relationship analysis were applied to evaluate the method. Results showed that with the proposed segmentation method, the correlation between reference GFR value and experimental value was quite well.

\section{Materials}

The dataset was collected from Tianjin Medical University General Hospital.

\subsection{Swine subjects}

The subjects were the swines (4 months-old pigs, weights $19.5-25 \mathrm{Kg}$ ) and 11 kidneys were included. The reference GFR was obtained from 99mTc-DTPA renal scintigraphy [10,19,20]. All subjects underwent 99mTc-DTPA scintigraphy first and gadopentetate dimeglumine (Gd-DTPA) dynamic contrastenhanced MR examinations afterwards. 


\subsection{Imaging protocol}

In this study, GE 3.0 T MRI equipment was used with the body phase array coil and 3D liver acceleration volume acquisition (LAVA). The scan range included abdominal aorta and kidneys for all the subjects. Based on a published paper [8], the low dose contrast agent was used to avoid underestimating the concentration of the contrast agent.

First, a non-contrast scan was performed, then an intravenous bolus of $0.04 \mathrm{mmol} / \mathrm{kg}$ of Gd-DTPA (which was produced by Bayer Schering Pharma, Germany) was given on ear with flow velocity at $3.0 \mathrm{ml} / \mathrm{s}$, and afterwards, the abdominal area was scaned 16 times repeatedly at an interval of 3 seconds. During the process of scan, a breath holding method was employed. The scan parameters were as follows: TR was $4.7 \mathrm{~ms}$, TE was $1.9 \mathrm{~ms}$, TI was $5.0 \mathrm{~ms}$, the flip angle was $15^{\circ}$, the planar matrix was $256 \times$ 256 , FOV was $30 \mathrm{~cm} \times 30 \mathrm{~cm}$, the slice thickness was $3.0 \mathrm{~mm}$, the pixel resolution was $1.372 \times 1.372$.

\section{Methods}

This paper proposed a method to segment the renal vascular compartment. Detailed steps can be described as follows.

\subsection{Signal conversion}

The Gd-DTPA is the most common contrast agent in MR contrast-enhanced scan. It can be filtered free in kidneys like Inulin. Most researches [21-24] agree that signal change and Gd-DTPA concentration have a linear relationship. Especially low dose of contrast media can ensure that the signal loss associated with concentrated gadolinium chelates can be avoided and make MR renography compatible with routine clinical contrast-enhanced imaging of the kidneys and renal arteries $[9,25]$. However, some researches [26,27] think differently on this conversion formula. In this paper, we adopted this linear conversion formula.

The conversion formula is:

$$
c=p \times \frac{S-S_{0}}{S_{0}}
$$

Where $c$ is the concentration of Gd-DTPA, $p$ is a constant and can be offset during calculation, $S$ is signal intensity after enhanced and $S_{0}$ is original signal intensity.

\subsection{Registration}

A proper registration step is critical in this procedure. Although a breath-hold method was adopted when performing the scan, the pig may have some respiratory motion, intestinal peristalsis, or body movement. Those motions can cause errors when using subtraction method between different phase images. Hence, it is necessary to make registration among images of the DCE-MRI time series to avoid the respiratory movement and other influence. Various registration algorithms have been used to solve this kind of problems. Affine registration [28] was used to correct the motions. Song et al. [29] proposed an automated 3D registration based on wavelet and Fourier transforms. Rohfling et al. [30] used intensity-based non-rigid registration to correct liver motion during respiratory cycle which used Bspline deformation model and mutual information. In order to reduce the deformation of kidney, we applied a B-spline registration frame on the time series, and used mutual information to assess the similarity. Because the MRR was 3D data, the registration is a time consuming work. In order to have an efficient calculation, a local registration was applied with the target only on the single kidney. 


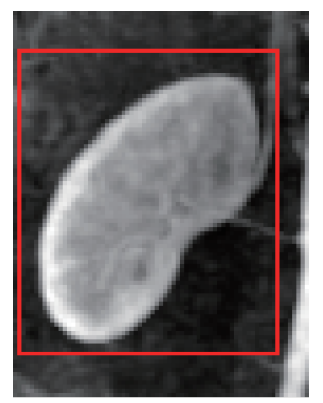

Fig. 1. The result of applying MIP and location box.

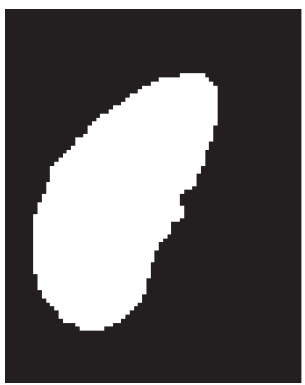

Fig. 2. The result of kidney segmentation.

\subsection{Segmentation of the whole kidney}

For the convenient of subsequent analysis and to reduce the unnecessary calculation, a mask of the kidney is needed. First of all, two phases scan images were selected. One was the non-contrast scan phase and the other was the phase with the cortex enhanced brightly. Then the Gussian filter was employed on these images. After that, the subtraction method was used between these two series images.

Secondly, maximum intensity projection technique (MIP) was applied to the subtraction result. In this procedure, the MIP technique can distinguish the foreground from background, which can help to locate the kidney position. Then, a rectangular box can be used to locate the kidney region. Showed in Fig. 1.

Thirdly, based on the MIP result, Ostu threshold method and some morphological methods were employed to get the binary image. The mask was shown in Fig. 2.

\subsection{Obtain the reconstructed feature image based on time density curves of each voxel}

The most challenging thing is that the renal vascular compartment is a functional unit, which means it is not a distinct anatomy structure. So it is not easy to be segmented from the original images. Therefore, a reconstructed image based on time density curve of each voxel was used to segment the vascular compartment.

MR renography is a kind of contrast enhanced MR scan data. In the process of continually scans of the body, the contrast agent flowed during each phase of the scan, and the signal intensity changed correspondingly. First the contrast agent flowed into the vascular compartment, and then flowed into the other compartments. The feature of each voxel's time density curve is quite different. By analyzing this, the vascular compartment can be distinguished from the other compartments. In Fig. 3, the pictures in the left column were the enhanced images. The red points marked on the images denote three different positions in the kidney. In the right column, the figures showed the corresponding time density curves of the voxel with red markers. These curves are also called as TDC curves. It can be seen from Fig. 3 that the peak time of each curve is quite different. This is the key to discriminate different compartment.

For each voxel in the kidney, its TDC curve can be obtained, and the peak time of the curve can also be calculated. Using these peak times we can create a new 3D feature images. Figure 4 showed the reconstructed image. In this image, the darker area means the contrast agent flowed faster and the lighter area means it flowed slower. 

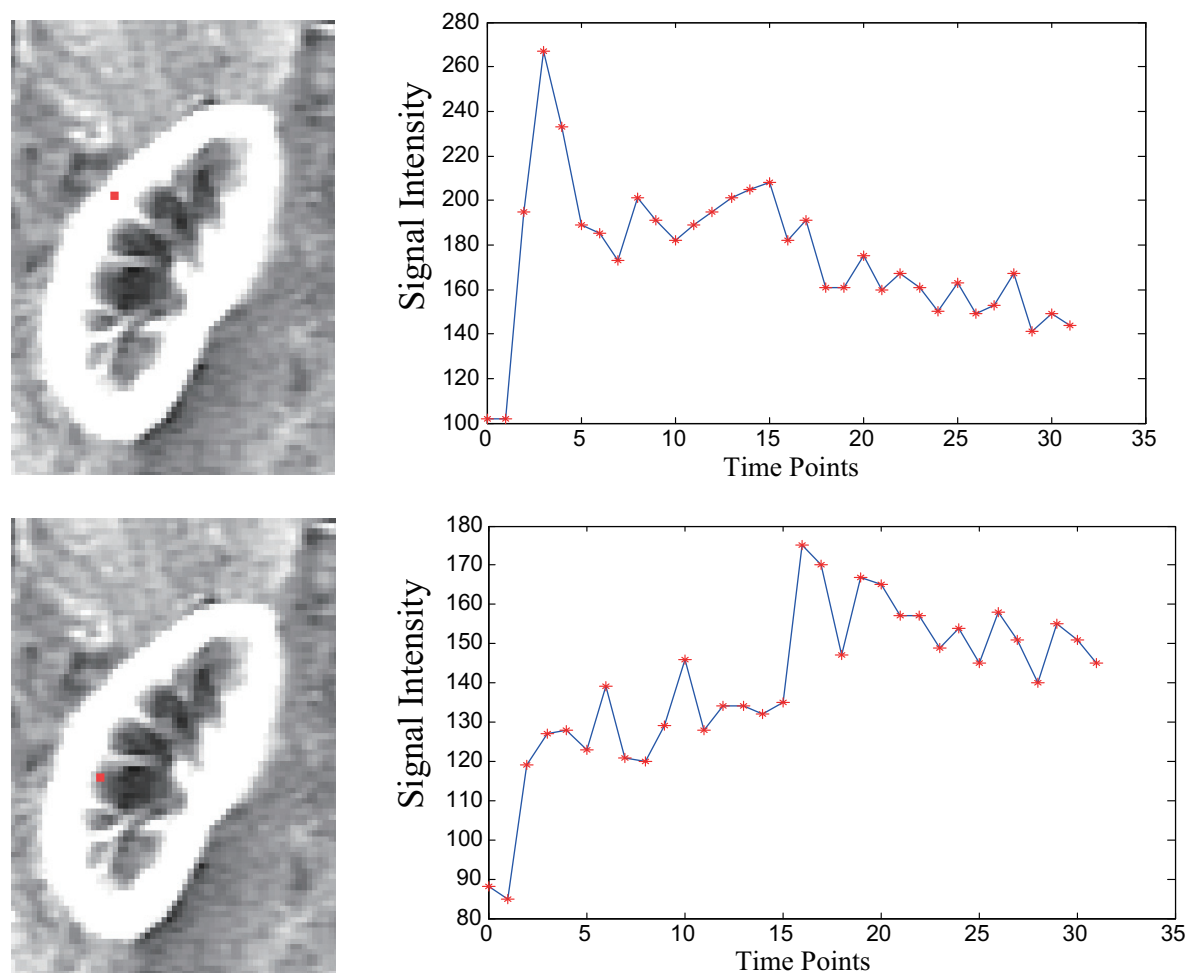

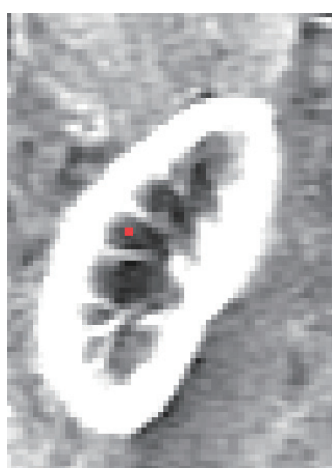

(a)

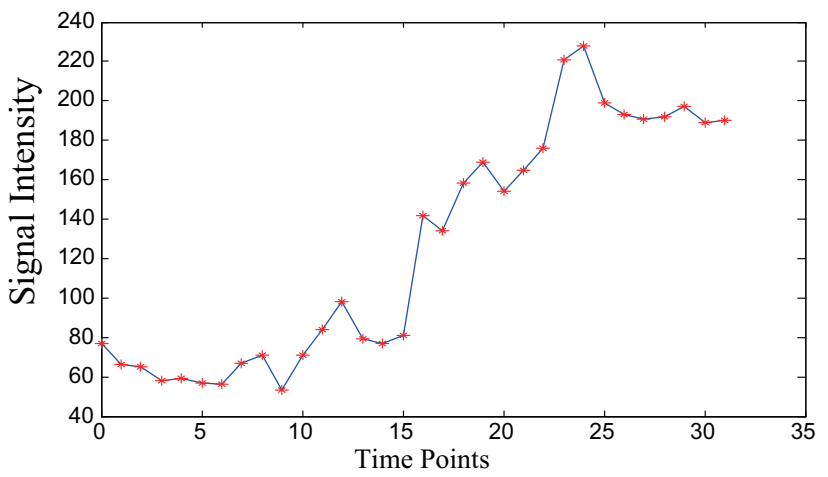

(b)

Fig. 3. (a) Enhanced kidney images with three markers on differernt positions; (b) Corresponding TDC curves of each marked point in red.

\subsection{Segment vascular compartment from the reconstructed feature image}

In reconstructed image, the Otsu threshold method was used to segment the vascular compartment. To reduce the computation and avoid the influence of the outside region of the kidney, the kidney mask obtained in Section 3.3 was used. Inside the kidney, the histogram of the gray value distribution was shown in Fig. 5.

According to Fig. 5, the Otsu threshold method can be used to segment the vascular compartment in addition with some morphological methods. 
Table 1

Reference GFR and model derived GFR of all subjects

\begin{tabular}{ccc}
\hline Subjects number & Reference GFR & Model derived GFR \\
\hline 1 & 41.100 & 37.510 \\
2 & 30.900 & 28.030 \\
3 & 46.500 & 37.050 \\
4 & 56.500 & 60.640 \\
5 & 42.400 & 31.555 \\
6 & 31.700 & 31.235 \\
7 & 30.900 & 27.340 \\
8 & 20.900 & 26.025 \\
9 & 33.500 & 29.825 \\
10 & 19.800 & 30.920 \\
11 & 74.900 & 73.525 \\
\hline
\end{tabular}

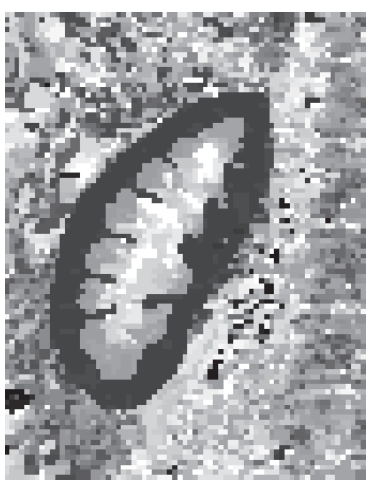

Fig. 4. The result of reconstruction image.

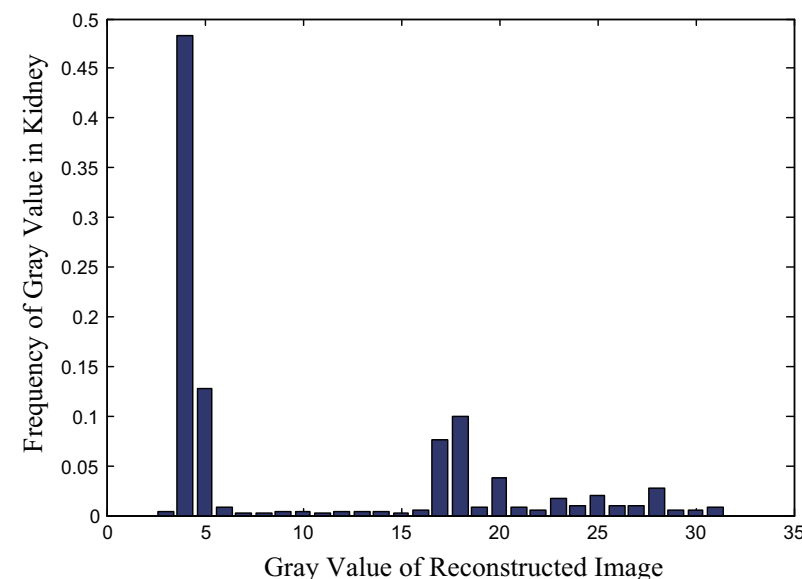

Fig. 5. The histogram of the gray value distribution in kidney of reconstructed image.

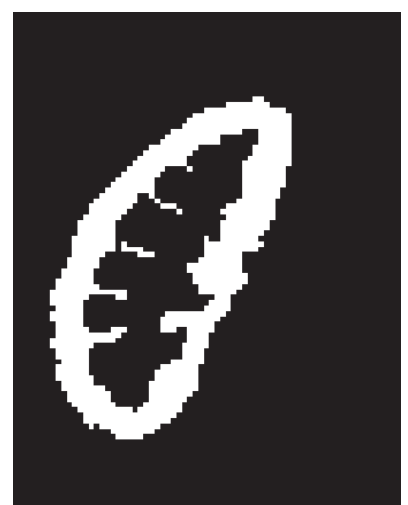

Fig. 6. The vascular compartment segmentation result.

\section{Results}

The final vascular compartment segmentation result was shown in Fig. 6.

In this study, a two compartments model with Patlak-Rutland technique [22] was applied to evaluate the effectiveness of the vascular compartment segmentation. The formula is showed as follow.

$$
K(t)=v_{b} \times b(t)+c \times \int_{0}^{t} b(u) d u
$$

Where $K(t)$ denotes the amount of Gd-DTPA in one kidney, $v_{b}$ denotes the volume of the vascular compartment, $b(t)$ means the signal change in vascular compartment and $c$ is GFR.

A total of 11 kidneys were tested with the algorithm we proposed. The reference GFR (golden standard) and model derived GFR (with the proposed segmentation method) were displayed in Table 1. 


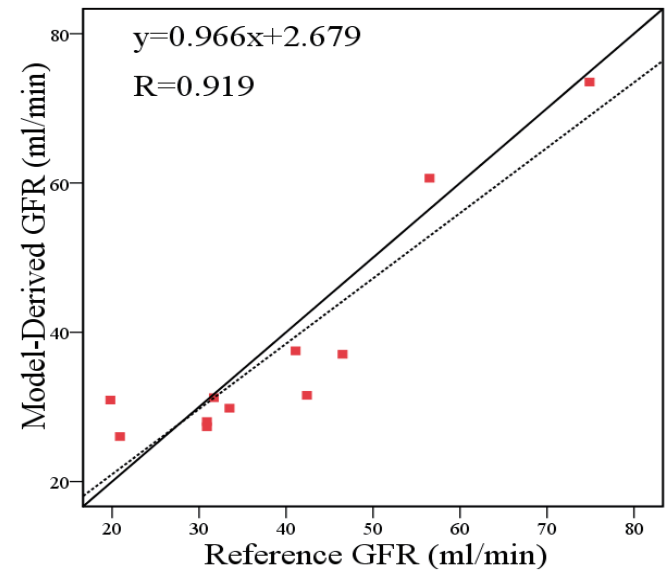

Fig. 7. Model derived GFR versus reference GFR.

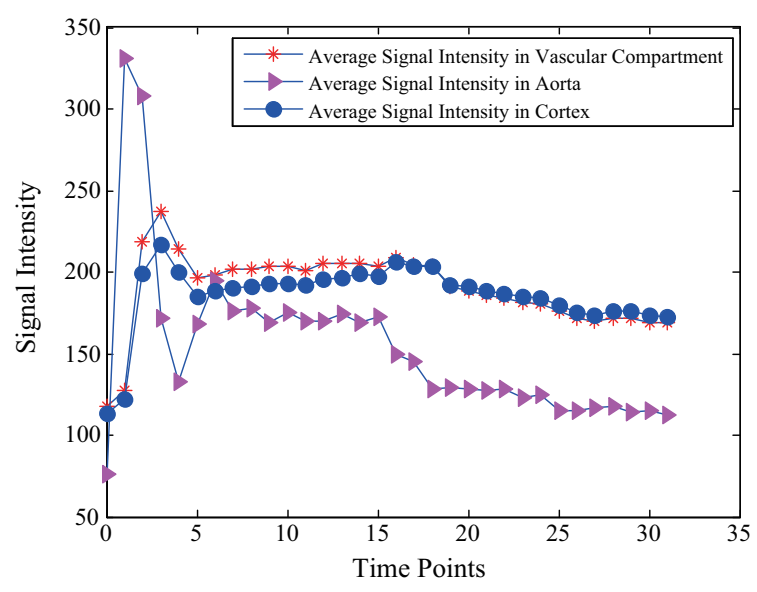

Fig. 8. Three curves of average signal intensity in vascular compartment, aorta, and cortex respectively.

\section{Discussion}

In this study, GFR obtained from scintigraphy was regarded as the reference value. Table 1 showed the reference GFR ranged from $19.8 \mathrm{ml} / \mathrm{min}$ to $74.9 \mathrm{ml} / \mathrm{min}$. To evaluate the accuracy of model derived GFR, the linear regression analysis was employed. Figure 7 showed the regression analysis. The correlation between model derived GFR and reference GFR was good $(R=0.919, P<0.00006)$. The equation of linear regression is $y=0.966 \cdot x+2.679$.

In Eq. (2), $b(t)$ denotes the concentration in vascular space. However, some researches [30-34] used the concentration in abdominal aorta to replace $b(t)$, which obviously brings a big influence on calculating the parameters [19]. Some papers used coronal plane sampling [7,25] and postprocessing corrections and population-averaged AIF curves [35]. Figure 8 showed three kinds of curves which were generated from vascular compartment, the aorta, and the renal cortex respectively. The difference can be found clearly.

From Fig. 8, it can be seen that the aorta curve had a sharp peak at time point 2 and drop rapidly at time point 4 . However, the cortex curve and vascular curve had a lower peak at time point 4 , and cortex curve was a little higher than aorta curve before time point 15 .

From the view of anatomy structure in kidney, the vascular compartment should be included both in cortex and medulla. In the paper of Zhang et al. [10], the ratio of vascular compartment inside cortex is $0.77 \pm 0.12$. That means the curve of vascular compartment should be consistent with the cortex curve, and higher than it a little bit.

\section{Conclusion}

Segmentation of vascular compartment is a very important part to assess renal function. Since the existing methods can not segment the vascular compartment, it has been assumed that the concentration of contrast agent in aorta is the same as it in the vascular compartment. However, this substitution can bring inaccurate factors to calculate the renal functional parameters.

In this paper, we proposed a novel method to segment the vascular space. The novelty of this method was to segment vascular compartment from a reconstructed image instead of original image. It used 
concentration curve of voxels to find the features that can distinguish vascular space from the others. This method used multi- phase scan images, and for a given kidney data, we can always get the same segmentation result.

According to the correlation and linear regression analysis, we can see that the correlation between reference GFR and model derived GFR (with the vascular compartment segmentation by our method) is $0.919(P<0.00006)$. This means that the method we proposed in vascular compartment segmentation can benefit the renal function analysis in a way.

\section{Acknowledgements}

This project was financially supported by National Natural Science Foundation of China (No. 612010 53, No. 81171316) and the Fundamental Research Funds for the Central Universities (N120419001).

\section{References}

[1] D. Baumann and M. Rudin, Quantitative assessment of rat kidney function by measuring the clearance of the contrast agent Gd (DOTA) using dynamic MRI, Magnetic Resonance Imaging, vol. 18, pp. 587-595,6, 2000.

[2] D. Laurent, K. Poirier, J. Wasvary, and M. Rudin, Effect of essential hypertension on kidney function as measured in rat by dynamic MRI, Magn Reson Med, vol. 47, pp. 127-34, Jan 2002.

[3] N. Hackstein, H. Kooijman, S. Tomaselli, and W.S. Rau, Glomerular filtration rate measured using the Patlak plot technique and contrast-enhanced dynamic MRI with different amounts of gadolinium-DTPA, J Magn Reson Imaging, vol. 22, pp. 406-14, Sep 2005.

[4] N. Hackstein, J. Heckrodt, and W.S. Rau, Measurement of single-kidney glomerular filtration rate using a contrastenhanced dynamic gradient-echo sequence and the Rutland-Patlak plot technique, Journal of Magnetic Resonance Imaging, vol. 18, pp. 714-725, 2003.

[5] C.S. Patlak, R.G. Blasberg, and J.D. Fenstermacher, Graphical evaluation of blood-to-brain transfer constants from multiple-time uptake data, J Cereb Blood Flow Metab, vol. 3, pp. 1-7, 1983.

[6] M.D. Rutland, A single injection technique for subtraction of blood background in 131I-hippuran renograms, The British Journal of Radiology, vol. 52, pp. 134-137, 1979.

[7] D.L. Buckley, A.E. Shurrab, C.M. Cheung, A.P. Jones, H. Mamtora, and P.A. Kalra, Measurement of single kidney function using dynamic contrast-enhanced MRI: comparison of two models in human subjects, J Magn Reson Imaging, vol. 24, pp. 1117-23, Nov 2006.

[8] L. Annet, L. Hermoye, F. Peeters, F. Jamar, J.P. Dehoux, and B.E. Van Beers, Glomerular filtration rate: assessment with dynamic contrast-enhanced MRI and a cortical-compartment model in the rabbit kidney, J Magn Reson Imaging, vol. 20, pp. 843-9, Nov 2004.

[9] V.S. Lee, H. Rusinek, L. Bokacheva, A.J. Huang, N. Oesingmann, Q. Chen et al., Renal function measurements from MR renography and a simplified multicompartmental model, American Journal of Physiology-Renal Physiology, vol. 292, pp. F1548-F1559, 2007.

[10] J.L. Zhang, H. Rusinek, L. Bokacheva, L.O. Lerman, Q. Chen, C. Prince et al., Functional assessment of the kidney from magnetic resonance and computed tomography renography: impulse retention approach to a multicompartment model, Magnetic Resonance in Medicine, vol. 59, pp. 278-288, 2008.

[11] L. Hermoye, L. Annet, P. Lemmerling, F. Peeters, F. Jamar, P. Gianello et al., Calculation of the renal perfusion and glomerular filtration rate from the renal impulse response obtained with MRI, Magn Reson Med, vol. 51, pp. 1017-25, May 2004.

[12] P.-H. Vivier, P. Storey, H. Rusinek, J.L. Zhang, A. Yamamoto, K. Tantillo et al., Kidney function: glomerular filtration rate measurement with MR renography in patients with cirrhosis, Radiology, vol. 259, pp. 462-470, 2011.

[13] H. Rusinek, Y. Boykov, M. Kaur, S. Wong, L. Bokacheva, J.B. Sajous et al., Performance of an automated segmentation algorithm for 3D MR renography, Magn Reson Med, vol. 57, pp. 1159-67, Jun 2007.

[14] Y. Boykov and O. Veksler, Graph cuts in vision and graphics: Theories and applications, in Handbook of mathematical models in computer vision, ed: Springer, pp. 79-96, 2006.

[15] Y. Boykov and G. Funka-Lea, Graph cuts and efficient ND image segmentation, International Journal of Computer Vision, vol. 70, pp. 109-131, 2006. 
[16] A.B. Chapman, L.M. Guay-Woodford, J.J. Grantham, V.E. Torres, K.T. Bae, D.A. Baumgarten et al., Renal structure in early autosomal-dominant polycystic kidney disease (ADPKD): The Consortium for Radiologic Imaging Studies of Polycystic Kidney Disease (CRISP) cohort1, Kidney international, vol. 64, pp. 1035-1045, 2003.

[17] S.W. van den Dool, M.N. Wasser, J.W. de Fijter, J. Hoekstra, and R.J. van der Geest, Functional renal volume: quantitative analysis at gadolinium-enhanced $\mathrm{mr}$ angiography - feasibility study in healthy potential kidney donors 1 , Radiology, vol. 236, pp. 189-195, 2005.

[18] J.A. de Priester, A.G.H. Kessels, E.L.W. Giele, J.A. den Boer, M.H.L. Christiaans, A. Hasman et al., MR renography by semiautomated image analysis: performance in renal transplant recipients, Journal of Magnetic Resonance Imaging, vol. 14, pp. 134-140, 2001.

[19] L. Bokacheva, H. Rusinek, J.L. Zhang, Q. Chen, and V.S. Lee, Estimates of glomerular filtration rate from MR renography and tracer kinetic models, Journal of Magnetic Resonance Imaging, vol. 29, pp. 371-382, 2009.

[20] J. Mørkenborg, M. Pedersen, F.T. Jensen, H. Stødkilde-Jørgensen, J.C. Djurhuus, and J. Frøkiær, Quantitative assessment of Gd-DTPA contrast agent from signal enhancement: an in-vitro study, Magnetic Resonance Imaging, vol. 21, pp. 637$643,7,2003$.

[21] V.S. Lee, H. Rusinek, G. Johnson, N.M. Rofsky, G.A. Krinsky, and J.C. Weinreb, MR renography with low-dose gadopentetate dimeglumine: feasibility 1, Radiology, vol. 221, pp. 371-379, 2001.

[22] G.L. Wolf, B. Hoop, J.A. Cannillo, J.A. Rogowska, and E.F. Halpern, Measurement of renal transit of gadopentetate dimeglumine with echo-planar MR imaging, Journal of Magnetic Resonance Imaging, vol. 4, pp. 365-372, 1994.

[23] R.W. Katzberg, M.H. Buonocore, M. Ivanovic, C. Pellot-Barakat, J.M. Ryan, K. Whang et al., Functional, dynamic, and anatomic mr urography: feasibility and preliminary findings, Academic Radiology, vol. 8, pp. 1083-1099,11, 2001.

[24] J. Taylor, P.E. Summers, S.F. Keevil, A.M. Saks, J. Diskin, P.J. Hilton et al., Magnetic resonance renography: optimisation of pulse sequence parameters and Gd-DTPA dose, and comparison with radionuclide renography, Magnetic Resonance Imaging, vol. 15, pp. 637-649, 1997.

[25] V.S. Lee, H. Rusinek, M.E. Noz, P. Lee, M. Raghavan, and E.L. Kramer, Dynamic Three-dimensional MR renography for the measurement of single kidney function: initial experience 1, Radiology, vol. 227, pp. 289-294, 2003.

[26] H. Rusinek, V.S. Lee, and G. Johnson, Optimal dose of Gd-DTPA in dynamic MR studies, Magnetic Resonance in Medicine, vol. 46, pp. 312-316, 2001.

[27] L. Bokacheva, H. Rusinek, Q. Chen, N. Oesingmann, C. Prince, M. Kaur et al., Quantitative determination of Gd-DTPA concentration in T1-weighted MR renography studies, Magnetic Resonance in Medicine, vol. 57, pp. 1012-1018, 2007.

[28] G.A. Buonaccorsi, J.P.B. O’Connor, A. Caunce, C. Roberts, S. Cheung, Y. Watson et al., Tracer kinetic model-driven registration for dynamic contrast-enhanced MRI time-series data, Magnetic Resonance in Medicine, vol. 58, pp. 10101019, 2007.

[29] T. Song, V.S. Lee, Q. Chen, H. Rusinek, and A.F. Laine, An automated three-dimensional plus time registration framework for dynamic MR renography, Journal of Visual Communication and Image Representation, vol. 21, pp. 1-8, 2010.

[30] T. Rohlfing, C.R. Maurer, W.G. O'Dell, and J. Zhong, Modeling liver motion and deformation during the respiratory cycle using intensity-based nonrigid registration of gated MR images, Medical Physics, vol. 31, pp. 427-432, 2004.

[31] J.P. Vallée, F. Lazeyras, H.G. Khan, and F. Terrier, Absolute renal blood flow quantification by dynamic MRI and GdDTPA, European Radiology, vol. 10, pp. 1245-1252, 2000/07/01 2000.

[32] H.J. Michaely, S.O. Schoenberg, N. Oesingmann, C. Ittrich, C. Buhlig, D. Friedrich et al., Renal artery stenosis: functional assessment with dynamic MR perfusion measurements - feasibility study 1, Radiology, vol. 238, pp. 586-596, 2006.

[33] X. Montet, M.K. Ivancevic, J. Belenger, M. Jorge-Costa, S. Pochon, A. Pechère et al., Noninvasive measurement of absolute renal perfusion by contrast medium-enhanced magnetic resonance imaging, Investigative Radiology, vol. 38, pp. 584-592, 2003.

[34] S.P. Sourbron, H.J. Michaely, M.F. Reiser, and S.O. Schoenberg, MRi-measurement of perfusion and glomerular filtration in the human kidney with a separable compartment model, Investigative Radiology, vol. 43, pp. 40-48 10.1097/RLI.0b013e31815597c5, 2008.

[35] G.J. Parker, C. Roberts, A. Macdonald, G.A. Buonaccorsi, S. Cheung, D.L. Buckley et al., Experimentally-derived functional form for a population-averaged high-temporal-resolution arterial input function for dynamic contrast-enhanced MRI, Magn Reson Med, vol. 56, pp. 993-1000, Nov 2006. 